\title{
On the distribution of inverses modulo $p$ (II)
}

\author{
by \\ Wenpeng Zhang (Shaanxi)
}

1. Introduction. Let $p$ be an odd prime. For each integer $a$ with $0<$ $a<p$, we define $\bar{a}$ by the congruence equation $a \bar{a} \equiv 1 \bmod p$ and $0<\bar{a}<p$. For any fixed positive integer $k$ and any fixed real number $0<\delta<1$, Professor Andrew Granville had proposed to study the limit distribution properties of

$$
\frac{1}{p-1} \sum_{\substack{a=1 \\|a-\bar{a}|<\delta p}}^{p-1} 1 .
$$

The author [3] completely solved this problem, and obtained a sharp asymptotic formula. That is, we proved that

$$
\sum_{\substack{a=1 \\|a-\bar{a}|<\delta p}}^{p-1} 1=\delta(2-\delta) p+O\left(p^{1 / 2} \ln ^{2} p\right) .
$$

In this paper, as a generalization of [3], we study the distribution properties of $\left|p\left\{a^{k} / p\right\}-p\left\{\bar{a}^{k} / p\right\}\right|$, and obtain a general asymptotic formula, where $\{x\}=x-[x],[x]$ denotes the greatest integer not exceeding $x$. In fact, we use the J. H. H. Chalk and R. A. Smith's deep result [2], which is based on E. Bombieri's work on exponential sums [1], and the estimates for trigonometric sums to prove the following more general conclusion:

THEOREM. Let $p$ be an odd prime. Then for any fixed positive integer $k$ and real number $0<\delta<1$, we have the asymptotic formula

$$
\sum_{\substack{a=1 \\\left|\left\{a^{k} / p\right\}-\left\{\bar{a}^{k} / p\right\}\right|<\delta}}^{p-1} 1=\delta(2-\delta) p+O_{k}\left(p^{1 / 2} \ln ^{2} p\right),
$$

where $O_{k}$ means that the $O$-constant depends only on $k$.

2000 Mathematics Subject Classification: 11N69, 11L05.

Key words and phrases: general Kloostermann's sums, distribution of inverses.

This work is supported by the N.S.F. and the P.N.S.F. of P.R. China. 
From this theorem we may immediately deduce the following

COROllary. Let $p$ be an odd prime, $k$ be any fixed positive integer. Then for any fixed real number $0<\delta<1$, we have the limit distribution formula

$$
\lim _{p \rightarrow \infty} \frac{1}{p} \sum_{\substack{a=1 \\\left|\left\{a^{k} / p\right\}-\left\{\bar{a}^{k} / p\right\}\right|<\delta}}^{p-1} 1=\delta(2-\delta) .
$$

REMARK. Let $F_{p}^{*}$ denote the multiplicative group formed by nonzero residue classes mod $p$. It is clear that the $k$-powers of nonzero residue classes $\bmod p$ form a multiplicative subgroup, say $U_{k}$, of $F_{p}^{*}$. If $k$ and $p-1$ are relatively prime, then $U_{k}$ is the full group $F_{p}^{*}$ and the result of our theorem reduces exactly to the case $k=1$, which was investigated in [3]. The new feature in this paper is when $(k, p-1)=d>1$ in which case $U_{k}=U_{d}$ is a proper subgroup of $F_{p}^{*}$. Thus the results of the present paper can be interpreted as results on the distribution of inverses inside a subgroup of small index in $F_{p}^{*}$.

2. Some lemmas. To prove the Theorem, we need several lemmas.

Lemma 1. Let $f, g$ be polynomials in $F_{p}[x, y]$ and suppose that

(a) $f(x, y)$ is absolutely irreducible in $F_{p}[x, y]$,

(b) $g(x, y) \not \equiv c(\bmod f(x, y))$ in $F_{p}[x, y]$ for any integer $c$.

Then we have the estimate

$$
\sum_{\substack{a=1 \\(a, b) \equiv 0(\bmod p)}}^{p} e\left(\frac{g(a, b)}{p}\right) \ll\left(d_{1}^{2}-3 d_{1}+2 d_{1} d_{2}\right) p^{1 / 2}+d_{1}^{2}
$$

for all primes $p$, where $F_{p}[x, y]$ denotes the set of all polynomials with coefficients in the residue systems modulo $p, d_{1}=d(f)$ and $d_{2}=d(g)$ are the degrees of $f$ and $g$ in $F_{p}[x, y]$, and $e(y)=e^{2 \pi i y}$.

Proof. See [2], Theorem 2.

Lemma 2. Let $p$ be an odd prime, $m$ and $n$ be integers. Then for any fixed positive integer $k$, we have the estimate

$$
\sum_{a=1}^{p-1} e\left(\frac{m a^{k}+n \bar{a}^{k}}{p}\right) \ll_{k} p^{1 / 2}(m, n, p)^{1 / 2},
$$

where $(m, n, p)$ denotes the greatest common divisor of $m, n$ and $p$.

Proof. It is clear that the assertion is true if $p \mid m$ and $p \mid n$. So without loss of generality we can assume $(m, n, p)=1$. Take $f(x, y)=x y-1$ and $g(x, y)=m x^{k}+n y^{k}$ in Lemma 1 and note that $g(x, y) \not \equiv c(\bmod f(x, y))$ in 
$F_{p}[x, y]$ for any integer $c$ if $(m, n, p)=1$. Applying Lemma 1 we immediately get the estimate

$$
\begin{aligned}
\sum_{a=1}^{p-1} e\left(\frac{m a^{k}+n \bar{a}^{k}}{p}\right) & =\sum_{\substack{a=1 \\
a b \equiv 1(\bmod p)}}^{p} \sum_{b=1}^{p} e\left(\frac{m a^{k}+n b^{k}}{p}\right) \\
& =\sum_{\substack{a=1 \\
f(a, b) \equiv 0(\bmod p)}}^{p} e\left(\frac{g(a, b)}{p}\right) \sum_{k} p^{1 / 2} .
\end{aligned}
$$

This proves Lemma 2 .

Lemma 3. Let $p$ be an odd prime. Then for any fixed real number $0<$ $\delta<1$, we have the estimate

$$
\sum_{r=1}^{p-1} \sum_{s=1}^{p-1}\left|\sum_{\substack{c=1 \\|c-d|<\delta p}}^{p-1} \sum_{d=1}^{p-1} e\left(\frac{-r c-s d}{p}\right)\right|=O\left(p^{2} \ln ^{2} p\right) .
$$

Proof. First note the trigonometric identity

$$
\sum_{a=1}^{n} e(a x)=e\left(\frac{(n+1) x}{2}\right) \frac{\sin \pi n x}{\sin \pi x} .
$$

Applying (2) we have

$$
\begin{aligned}
\sum_{r=1}^{p-1} \sum_{s=1}^{p-1}\left|\sum_{\substack{c=1 \\
|c-d|<\delta p}}^{p-1} \sum_{d=1}^{p-1} e\left(\frac{-r c-s d}{p}\right)\right| & \\
\leq & 2 \cdot \sum_{r=1}^{p-1} \sum_{s=1}^{p-1}\left|\sum_{m=0}^{[\delta p]} \sum_{\substack{c=1 \\
c-d=m}}^{p-1} \sum_{d=1}^{p-1} e\left(\frac{-r c-s d}{p}\right)\right| \\
& =2 \cdot \sum_{r=1}^{p-1} \sum_{s=1}^{p-1}\left|\sum_{m=0}^{[\delta p]} \sum_{d=1}^{p-1-m} e\left(\frac{-r(d+m)-s d}{p}\right)\right| \\
& =2 \cdot \sum_{r=1}^{p-1} \sum_{s=1}^{p-1}\left|\sum_{m=0}^{[\delta p]} e\left(\frac{-r m}{p}\right) \sum_{d=1}^{p-1-m} e\left(\frac{-(r+s) d}{p}\right)\right| \\
& \ll \sum_{r=1}^{p-1}\left|\sum_{m=0}^{[\delta p]} e\left(\frac{-r m}{p}\right)(p-1-m)\right|
\end{aligned}
$$




$$
\begin{aligned}
& +\sum_{\substack{r=1 \\
r+s \neq p}}^{p-1} \sum_{\substack{s=1 \\
m=0}}^{p-1} \sum_{m=0}^{[\delta p]} e\left(\frac{-r m}{p}\right) e\left(\frac{-(r+s)}{p}\right) \frac{e\left(\frac{-(r+s)(p-1-m)}{p}\right)-1}{e\left(\frac{-(r+s)}{p}\right)-1} \mid \\
& \ll \sum_{r=1}^{p-1}\left|\sum_{m=0}^{[\delta p]} e\left(\frac{-r m}{p}\right)(p-1-m)\right|+\sum_{\substack{r=1 \\
r+s \neq p}}^{p-1} \sum_{\substack{p=1 \\
p-1}} \frac{1}{\left|e\left(\frac{-(r+s)}{p}\right)-1\right|} \\
& \quad \times\left|\sum_{m=0}^{[\delta p]} e\left(\frac{-r m-(r+s)(p-1-m)}{p}\right)-\sum_{m=0}^{[\delta p]} \in\left(\frac{-r m}{p}\right)\right| .
\end{aligned}
$$

Note the trigonometric sum estimate

$$
\sum_{m \leq M} m^{k} e(m x) \leq M^{k} \min \left(M, \frac{1}{|\sin \pi x|}\right) \quad \text { for } k \geq 0 .
$$

From (3) and (4) we get

$$
\begin{aligned}
& \sum_{r=1}^{p-1} \sum_{s=1}^{p-1} \mid \sum_{\substack{c=1 \\
|c-d|<\delta p}}^{p-1} \sum_{d=1}^{p-1} e\left(\frac{-r c-s d}{p}\right) \mid \\
& \ll \sum_{r=1}^{p-1} \frac{p}{\left|\sin \frac{\pi r}{p}\right|}+\sum_{\substack{r=1 \\
r+s \neq p}}^{p-1} \sum_{s=1}^{p-1} \frac{1}{\left|\sin \frac{\pi(r+s)}{p}\right|}\left[\frac{1}{\left|\sin \frac{\pi r}{p}\right|}+\frac{1}{\left|\sin \frac{\pi s}{p}\right|}\right] \\
& \ll \frac{1}{p-1} \ln p+\sum_{r=1}^{p-1} \frac{1}{\left|\sin \frac{\pi r}{p}\right|} \sum_{\substack{s=1 \\
s \neq p-r}}^{\left|\sin \frac{\pi(r+s)}{p}\right|} \\
& \ll p^{2} \ln ^{2} p .
\end{aligned}
$$

This proves Lemma 3.

3. Proof of the Theorem. In this section, we complete the proof of the Theorem. First note the trigonometric identity

and the identity

$$
\sum_{r=1}^{q} e\left(\frac{r n}{q}\right)= \begin{cases}q & \text { if } q \mid n \\ 0 & \text { if } q \nmid n,\end{cases}
$$

$$
\begin{aligned}
& \sum_{r=1}^{p-1} \sum_{s=1}^{p-1}\left[\sum_{\substack{a=1 \\
a b \equiv 1(\bmod p)}}^{p-1} \sum_{b=1}^{p-1} e\left(\frac{r \cdot p\left\{\frac{a^{k}}{p}\right\}+s \cdot p\left\{\frac{b^{k}}{p}\right\}}{p}\right)\right] \\
&=\sum_{r=1}^{p-1} \sum_{s=1}^{p-1}\left[\sum_{a=1}^{p-1} e\left(\frac{r \cdot a^{k}+s \cdot \bar{a}^{k}}{p}\right)\right] .
\end{aligned}
$$


From the estimates for trigonometric sums and Lemmas 2 and 3 we have

$$
\begin{aligned}
& \sum_{a=1}^{p-1} 1 \\
& \left|\left\{a^{k} / p\right\}-\left\{\bar{a}^{k} / p\right\}\right|<\delta \\
& =\sum_{\substack{a=1 \\
a b \equiv 1(\bmod p) \\
\left\{a^{k} / p\right\}-\left\{b^{k} / p\right\} \mid<\delta}}^{p-1} \sum_{\substack{b-1\\
}}^{p-1} 1 \\
& =\frac{1}{p^{2}} \sum_{r=1}^{p} \sum_{s=1}^{p} \sum_{\substack{a=1 \\
a b \equiv 1(\bmod p)}}^{p-1} \sum_{\substack{c=1 \\
|c-d|<\delta p}}^{p-1} \sum_{\substack{d=1 \\
\mid c-1}}^{p-1}\left(\frac{r\left(p\left\{\frac{a^{k}}{p}\right\}-c\right)}{p}\right) e\left(\frac{s\left(p\left\{\frac{b^{k}}{p}\right\}-d\right)}{p}\right) \\
& =\frac{1}{p^{2}} \sum_{r=1}^{p} \sum_{s=1}^{p}\left[\sum_{\substack{a=1 \\
a b \equiv 1(\bmod p)}}^{p-1} \sum_{\substack{p-1 \\
p}}^{p \cdot p\left\{\frac{a^{k}}{p}\right\}+s \cdot p\left\{\frac{b^{k}}{p}\right\}} \sum_{\substack{c=1 \\
|c-d|<\delta p}}^{p-1} \sum_{d=1}^{p-1} e\left(\frac{-r c-s d}{p}\right)\right.
\end{aligned}
$$

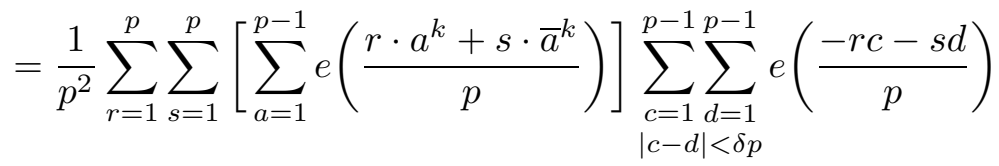

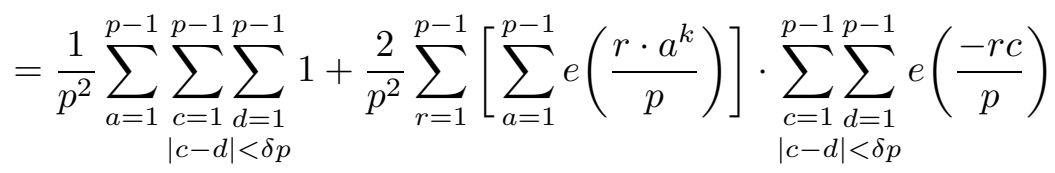

$$
\begin{aligned}
& +\frac{1}{p^{2}} \sum_{r=1}^{p-1} \sum_{s=1}^{p-1}\left[\sum_{a=1}^{p-1} e\left(\frac{r \cdot a^{k}+s \cdot \bar{a}^{k}}{p}\right)\right] \sum_{\substack{c=1 \\
|c-d|<\delta p}}^{p-1} \sum_{d=1}^{p-1} e\left(\frac{-r c-s d}{p}\right) \\
& =\frac{1}{p^{2}}(p-1)\left[2 \cdot \sum_{m=0}^{[\delta p]} \sum_{\substack{c=1 \\
c-d=m}}^{p-1} \sum_{\substack{d=1 \\
c-1}}^{p-1}\right]+O(1) \\
& +O_{k}\left(p^{-2+1 / 2} \cdot \sum_{r=1}^{p-1}\left|\sum_{\substack{c=1 \\
|c-d|<\delta p}}^{p-1} \sum_{d=1}^{p-1} e\left(\frac{-r c}{p}\right)\right|\right) \\
& +O_{k}\left(p^{-2+1 / 2} \cdot \sum_{r=1}^{p-1} \sum_{s=1}^{p-1}\left|\sum_{\substack{c=1 \\
|c-d|<\delta p}}^{p-1} \sum_{d=1}^{p-1} e\left(\frac{-r c-s d}{p}\right)\right|\right)
\end{aligned}
$$




$$
\begin{aligned}
= & \frac{1}{p^{2}}(p-1)\left[2 \cdot \sum_{m=0}^{[\delta p]}(p-1-m)\right]+O(1) \\
& +O_{k}\left(p^{-2+1 / 2} \cdot \sum_{c=1}^{p-1}(\delta p+c) \cdot \frac{1}{\left|\sin \frac{\pi c}{p}\right|}\right)+O_{k}\left(p^{1 / 2} \ln ^{2} p\right) \\
= & \frac{1}{p^{2}}(p-1)\left[2 p(\delta p+1)-\delta^{2} p^{2}+O(p)\right]+O_{k}\left(p^{1 / 2} \ln ^{2} p\right) \\
= & p \delta(2-\delta)+O_{k}\left(p^{1 / 2} \ln ^{2} p\right) .
\end{aligned}
$$

This completes the proof of the Theorem.

Acknowledgments. The author express his gratitude to the referee for his very helpful and detailed comments.

\section{References}

[1] E. Bombieri, On exponential sums in finite fields, Amer. J. Math. 88 (1966), 71-105.

[2] J. H. H. Chalk and R. A. Smith, On Bombieri's estimate for exponential sums, Acta Arith. 18 (1971), 191-212.

[3] W. P. Zhang, On the distribution of inverses modulo n, J. Number Theory 61 (1996), 301-310.

Research Center for Basic Science

Xi'an Jiaotong University

Xi'an, Shaanxi, P.R. China

E-mail: wpzhang@nwu.edu.cn 